\title{
SOME PROPERTIES OF FC-GROUPS WHICH OCCUR AS AUTOMORPHISM GROUPS
}

\author{
JAY ZIMMERMAN
}

\begin{abstract}
We prove that if $G$ is a group such that Aut $G$ is a countably infinite torsion FC-group, then Aut $G$ contains an infinite locally soluble, normal subgroup and hence a nontrivial abelian normal subgroup. It follows that a countably infinite subdirect product of nontrivial finite groups, of which only finitely many have nontrivial abelian normal subgroups, is not the automorphism group of any group.
\end{abstract}

We are concerned with the question: What classes of torsion groups can occur as the full group of automorphisms Aut $G$ of a group $G$ ? Robinson [1] has shown that if Aut $G$ is a Černikov group (a finite extension of a radicable abelian group with the minimal condition), then Aut $G$ is finite. He has also shown that if Aut $G$ is a nilpotent torsion group, then Aut $G$ has finite exponent.

The case where $G$ is a group such that Aut $G$ is a countable torsion FC-group (finite conjugate) was examined in a previous paper [2]. It was shown that if $G$ is a group such that Aut $G$ is a countable torsion FC-group, then Aut $G$ has finite exponent if either (1) Aut $G$ has $\min -2$ or (2) $\pi$ (Aut $G$ ) is finite, where $\pi(H)$ is the set of all primes dividing the order of some torsion element of $H$. In addition, an example of a countable torsion FC-group of infinite exponent which occurred as an automorphism group was given to show that the theorem could not be improved. This example contains a nontrivial abelian normal subgroup. The question arises: Can we find an example which has no nontrivial abelian normal subgroups? We will answer this question in the negative.

THEOREM. Let $G$ be a group such that Aut $G$ is a countably infinite periodic FC-group. Then either

(a) Aut $G$ contains an infinite abelian normal subgroup $N$, or

(b) Aut $G$ contains an infinite, locally soluble, normal $\{2,3\}$-subgroup of bounded exponent and finite index.

In either case, Aut $G$ contains a nontrivial abelian normal subgroup.

Proof. Let $Q=G / C \cong \operatorname{Inn} G$, where $C$ is the center of $G$, and let $T$ be the torsion subgroup of $C$. It was proven in [2] that $Q$ and $T_{p}$ are finite for all primes $p$.

Let $q=|Q|$ and let $p$ be any prime which does not divide $2 q$. Since $T_{p}$ is finite, we have $C=C_{1} \times T_{p}$. It is well known that since $|Q|$ and $\left|T_{p}\right|$ are relatively prime, $G$ splits over $T_{p}$. It follows that there exists a group $G_{1}$ containing $C_{1}$ such that

Received by the editors November 26, 1984 and, in revised form, January 20, 1985.

1980 Mathematics Subject Classification. Primary 20F28, 20E26.

Key words and phrases. Automorphism group, torsion FC-group, semisimple group. 
$G=G_{1} \times T_{p}$. Clearly, $T_{p}$ is characteristic in $G$. Hence we have the short exact sequence

$$
\operatorname{Hom}\left(G_{1}, T_{p}\right) \nrightarrow \text { Aut } G \rightarrow \text { Aut } G_{1} \times \text { Aut } T_{p} \text {. }
$$

Let $I$ be the set of all primes $p$ not dividing $2 q$ such that $T_{p} \neq 1$.

Case 1: $I$ is infinite. Define $M_{p}=\operatorname{Hom}\left(G_{1}, T_{p}\right) \triangleleft$ Aut $G$. Assume that $p \in I$. If $M_{p} \neq 1$, define $N_{p}=M_{p}$. If $M_{p}=1$, then Aut $G \cong$ Aut $G_{1} \times$ Aut $T_{p}$ and define $N_{p}=Z\left(\right.$ Aut $\left.T_{p}\right) \leqslant$ Aut $G$. Since the inversion automorphism on $T_{p}$ is contained in $Z$ (Aut $T_{p}$ ), the group $N_{p}$ is nontrivial. It is easily shown that $N=\left\langle N_{p} \mid p \in I\right\rangle$ is an abelian normal subgroup of Aut $G$ which is infinite.

Case 2: $I$ is finite. It follows that $T$ is finite and hence $C=F \times T$ for some torsion-free group $F$. In the proof of Lemma 7 and Theorem A in [2], it was shown that under these circumstances there exists a normal subgroup $N$ of Aut $G$ such that Aut $G / N$ is finite and $N$ is a $\{2,3\}$-group of finite exponent. Clearly, $N$ is a locally soluble, normal subgroup of Aut $G$. If $N$ is finite, then Aut $G$ is finite. However, since Aut $G$ is infinite, it must have an infinite locally soluble, normal subgroup $N$. Since Aut $G$ is a periodic FC-group, it is locally finite and normal. Hence Aut $G$ contains a finite normal subgroup which is soluble and therefore a nontrivial abelian normal subgroup.

Corollary 1. Let $G$ be a group such that Aut $G$ is a countably infinite periodic $F C$-group. If either Aut $G$ has infinite exponent or if it has no elements of order 2 or 3 , then Aut $G$ contains an infinite abelian normal subgroup.

COROLlARY 2. If among the countably infinite sequence of nontrivial finite groups $F_{i}$ there are only finitely many with a nontrivial soluble normal subgroup, then no subdirect product of the $F_{i}$ can be an automorphism group.

\section{REFERENCES}

1. D. J. S. Robinson, Infinite torsion groups as automorphism groups, Quart. J. Math. Oxford Ser. (2) 30 (1979), 351-364.

2. J. Zimmerman, Countable torsion FC-groups as automorphism groups, Arch. Math. 43 (1984), $108-116$.

Department of Mathematics, Michigan State University, East Lansing, Michigan 48824 